\title{
A patient with primary syphilis of the hand
}

\author{
G. A. J. DE KONING, F. B. BLOG, AND E. STOLZ \\ From the Department of Dermatology of the University Hospital Rotterdam-Dijkzigt, \\ The Netherlands
}

SUMMARY The case history of a man with primary syphilis of the right hand is described. This type of luetic condition nowadays is rare. The incidence of genital, anorectal, and extragenital primary syphilis in both men and women and in sailors presenting at the Venereological Outpatient Clinic of the Department of Dermatology of the University Hospital Rotterdam-Dijkzigt during a period of seven years was studied. In sailors and women only genital primary lesions were found, whereas in male civilians $6 \cdot 3 \%$ of cases of primary syphilis had anorectal lesions. The primary lesions in this study were different from those reported in earlier literature.

\section{Introduction}

There are now few reports of extragenital primary syphilis, other than anorectal primary lesions (Hollings, 1961; Samenius, 1968; Menda et al., 1971; Nazemi et al., 1975) and oropharyngeal primary syphlitic lesions (Vincenti, 1971; Fiumara and Berg, 1974).

Earlier reports (Willcox, 1964; Jadassohn, 1965) showed that extragenital primary lesions used to be seen more often, and lesions of the head represented $70 \%$ of all extragenital lesions (Hiemcke, 1935). Primary syphilis of the fingers, hands, and arms and anorectal lesions were seen less frequently.

Primary syphilis is seldom diagnosed in women, and there are few reports of women having genital primary lesions. During the last few years we have mainly seen men with genital and anorectal primary lesions in our clinic; primary syphilis is comparatively rare in women.

In 1976 we saw a patient with primary lesions on the fingers of his right hand. In view of the rarity of this form of primary syphilis we are presenting the case history.

We also checked our records to see how many new patients had visited our clinic during the last seven years and we compared the incidence of genital, anorectal, and other primary lesions. The patients were divided into three groups-men (civilians and sailors) and women.

Address for reprints: E. Stolz, Department of Dermatology, University Hospital Rotterdam-Dijkzigt, Rotterdam, Holland

Received for publication 8 June 1977
CASE HISTORY

A 28-year-old Turkish man presented at our Venereological Outpatient Clinic on 16 September 1976. He had been referred because he had ulcers of the right middle and little fingers that had failed to heal. The referring physician also reported to have seen diplococci in the methylene blue stained smear. The patient claimed to have incurred the ulcers in a fight with another Turk, by whom he had been bitten on his middle and little fingers. One month before coming to our clinic the patient had had manual sexual contact with the vulva and vagina of an otherwise unknown woman. He denied other sexual contact during the previous three months.

On examination, there were three exudative ulcers on the middle finger (Figure) and one on the little finger of the right hand. The ulcers were firm and painless on palpation. In the right axilla a firm, painless lymph node was palpated. Further examination revealed no abnormality.

\section{LABORATORY TESTS}

Erythrocyte sedimentation rate $4 \mathrm{~mm}$ in the first hour, haemoglobin $10.3 \mathrm{mmol} / 1$, leucocytes (WBC) $5.6 \times 10^{\circ} / 1$. In the darkfield preparation of the exudate from the ulcers viable, motile treponemes were seen. Their length, appearance, and movements were characteristic of Treponema pallidum. The serological results were: Kölmer reaction negative, Venereal Disease Research Laboratory (VDRL) test positive, rapid plasma reagin (RPR) test positive, Treponema pallidum immobilisation (TPI) test positive, fluorescent treponemal antibody absorption (FTA/ABS) test positive $(++)$, Treponema 


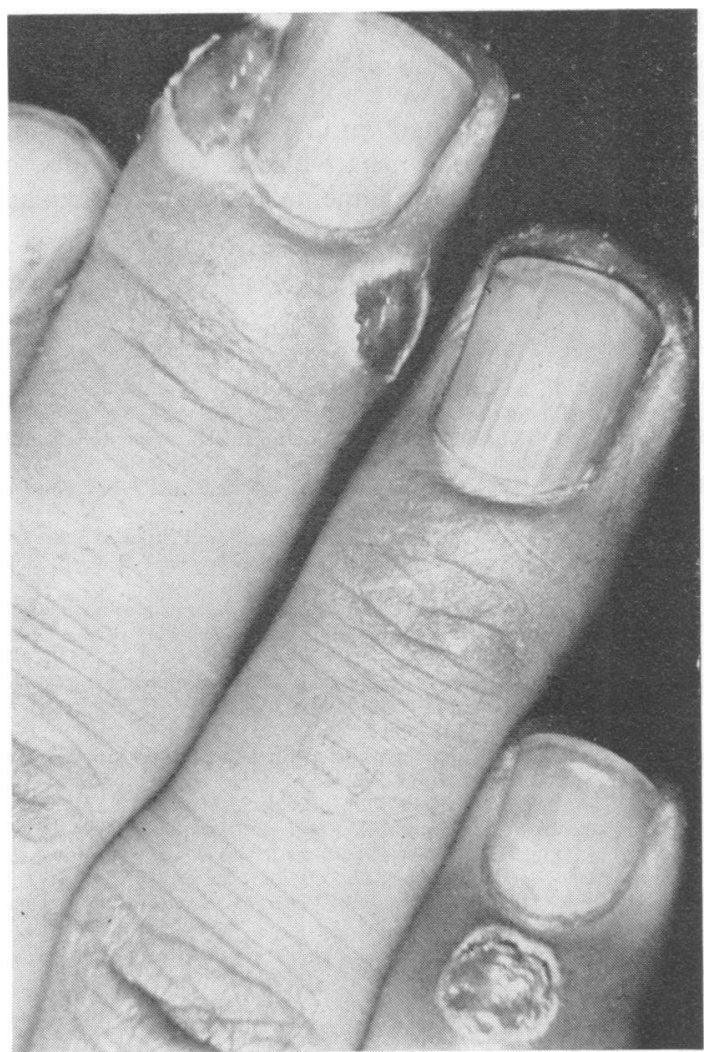

Figure Primary syphilitic lesions

pallidum haemagglutination assay (TPHA) negative.

In the Gram stain no Gram-negative diplococci and no Gram-negative rods resembling Haemophilus ducreyi were seen, but we did see many Grampositive cocci. A culture of the pus showed a growth of Staphylococcus aureus and Streptococcus haemolyticus. A selective culture for gonococci was negative. Herpes simplex virus could not be cultured from the ulcers.

The complement-fixation tests for herpes simplex virus and for varicellae zoster virus were $1: 32$ and $1: 4$ respectively. Selective cultures for gonococci from oropharyngeal and from urethral swabs were negative. The two-glass urine test showed no urethritis. Examination of the partner was not possible.

Since typical looking $T$. pallidum had been found in the darkfield preparation, and as the patient had positive serological results, we diagnosed primary syphilis of the right hand.

The patient was treated with a series of 18 injections of 600000 units of penicillin-aluminium monostearate three times a week: a total of $10 \cdot 8$ megaunits. There was no Herxheimer reaction.

Soon after the start of treatment the ulcers healed with the formation of scar tissue.

When this patient was followed-up two weeks later the complement-fixation tests for herpes simplex virus and for varicellae zoster virus were $1: 16$ and $1: 4$ respectively.

After six weeks the following serological results were found: Kölmer reaction negative, VDRL negative, RPCF negative, TPI negative, FTA/ABS positive $(+)$. After 12 weeks the Kölmer reaction, VDRL, and RPCF were still negative, TPI was not performed, and the FTA/ABS was positive $(+)$.

The changes in the results of the serological tests after treatment support the diagnosis of primary syphilis.

\section{Review of our records at the clinic}

From 1970 to 1976, 20280 new patients were seen in our Venereological Outpatient Department. Of these patients $8207(40.5 \%)$ were male civilians, $7188(35.4 \%)$ were sailors, and $4885(24 \cdot 1 \%)$ were women. Of the male civilians, $287(3.5 \%$ of the total number of new male civilian patients) had primary syphilis. The percentages for sailors and women were $5.2 \%$ (377) and $0.7 \%$ (34) respectively. Of the male civilian population, $18(6.3 \%$ of the total number with primary syphilis) had an anorectal lesion and one $(0.3 \%$ of all primary cases) had an extragenital lesion. All other male civilians had genital lesions. The sailors and women all had primary lesions of the genitals.

It is important to note that all primary cases in women were in patients who were seen weekly as named contacts of male patients with infectious syphilis. These women had not been treated for epidemiological reasons.

\section{Discussion}

Primary syphilis was most often found in sailors $(5 \cdot 2 \%)$, slightly less often in male civilians $(3.5 \%)$, and rarely in women $(0.7 \%)$.

Extragenital primary syphilis used to be common, and was often seen accidentally, for example by surgeons or midwives (Willcox, 1964). From our material it was found that extragenital primary syphilis has become very rare.

Our interest in this particular patient was aroused by the referral letter from his family doctor, who thought this was a gonococcal infection. On inspection and palpation the ulcers and regional lymph nodes were firm and painless. For this reason a darkfield preparation was made which led us to the diagnosis. 
Nevertheless the diagnosis of syphilis would have been made as serological tests for syphilis are done routinely on all patients in our dermatological and venereological outpatient departments.

It is noteworthy, that no oropharyngeal primary syphilis was found in our patients. It is difficult to give a proper explanation for this. It is known that many of the patients who come to our outpatient department have a regular orogenital sexual contact. This is demonstrated by the high percentage of oropharyngeal gonorrhoea in patients with a gonococcal infection (about $4 \%$ in men and about $8 \%$ in women) (Stolz and Schuller, 1974). It is possible that the treatment leading to shorter asymptomatic infectiousness in early syphilis plays a role here.

Among our patients anorectal lesions were found only in homosexual men. As we do not have statistics on the percentage of homosexuals, we have no way of knowing whether the incidence of primary syphilis is higher in homosexuals than in heterosexuals.

The fact that primary syphilis was found only in women who had been named as the contacts of men with early infectious syphilis raises the question: Was treatment on epidemiological grounds necessary? However, these women had been clearly instructed not to have any further sexual contact during the period that they were being followed-up by us and, as far as we could find out, they had not caused any new infection. All those women with primary syphilis had negative serological results, in lipoidal as well as in treponemal tests, after adequate penicillin treatment.

\section{References}

Fiumara, N. J., and Berg, M. (1974). Primary syphilis in the ora cavity. British Journal of Venereal Diseases, 50, 463-464.

Hiemcke, H. J. Th. (1935). Multiple extragenitale primaire affecten. Nederlands Tijdschrift voor Geneeskunde, 79, 1085-1094.

Hollings, R. M. (1961). Syphilitic ulcers of the anus. Proceedings of the Royal Society of Medicine, 54, 730-731.

Jadassohn, J. (1965). Genitale und extragenitale Primär Affekte. In Handbuch der Haut - und Geschlechtskrankheiten, 6/2 A p. 886. Springer Verlag: Berlin.

Menda, R. K., Chulani, H. L., Yawalkar, S. J., and Kulkarni, B. S. (1971). Venereal disease of the anal region. Diseases of the Colon and Rectum, 14, 454-459.

Nazemi, M. M., Musher, D. M., Schell, R. F., and Milo, S. (1975). Syphilitic proctitis in a homosexual. Journal of the American Medical Association, 231, 389.

Samenius, B. (1968). Primary syphilis of the anorectal region. Diseases of the Colon and Rectum, 11, 462-465.

Stolz, E., and Schuller, J. (1974). Gonococcal oro- and nasopharyngeal infection. British Journal of Venereal Diseases, 50, 104-108.

Vincenti, N. H. (1971). A case of sero-positive primary syphilis of the tonsil. Journal of Laryngology and Otology, 85, 869-870.

Willcox, R. R. (1964). Textbook of Venereal Diseases and Treponematoses. Heinemann: London. 